\title{
Transtorno do desenvolvimento da coordenação e senso de auto-eficácia: implicações para a prática da terapia ocupacional
}

\section{Developmental coordination disorder and sense of self-efficacy: implications for occupational therapy's practice}

\author{
Beatriz A P. Galvãoํ, Naira Vassalo Lage', \\ Ana Amélia C. Rodrigues ${ }^{2}$
}

GALVÃO, B. A. P.; LAGE, N. V.; RODRIGUES, A. A. C. Transtorno do desenvolvimento da coordenação e senso de auto-eficácia: implicações para a prática da terapia ocupacional. Rev. Ter. Ocup. Univ. São Paulo, v.19, n. 1, p. 12-19, jan./abr. 2008.

RESUMO: No transtorno do desenvolvimento da coordenação há um prejuízo acentuado no desenvolvimento das habilidades motoras (grossas e/ou finas) de crianças, com impacto na participação em atividades típicas da infância. As conseqüências do transtorno também incluem restrição na interação social e baixo senso de auto-eficácia. Terapeutas ocupacionais, embora habilitados a avaliar e tratar crianças com esse perfil, aparentam desconhecer a importância do senso de auto-eficácia, assim como seus instrumentos de avaliação. Desta forma, o objetivo desta revisão é apontar a relação entre o desempenho motor e o senso de eficácia de crianças com TDC em idade escolar, bem como suas possíveis implicações para a prática da Terapia Ocupacional.

DESCRITORES: Transtorno das habilidades motoras. Auto-eficácia. Desempenho psicomotor. Criança. Terapia Ocupacional.

${ }^{1}$ Acadêmicas do $10^{\circ}$ período de Terapia Ocupacional da Universidade Federal de Minas Gerais (UFMG).

2 Terapeuta Ocupacional. Doutoranda em Ciências da Reabilitação na Universidade Federal de Minas Gerais (UFMG).

Endereço para correspondência: Beatriz de Arruda Pereira Galvão. Rua São Paulo, 1333 Apto. 512 -Centro, Belo Horizonte, MG. Cep: 30.170-131. E-mail: beatrizgalvao@gmail.com 
GALVÃO, B. A. P. et al. Transtorno do desenvolvimento. Rev. Ter. Ocup. Univ. São Paulo, v.19, n. 1, p. 12-19, jan./abr. 2008.

\section{INTRODUÇÃO}

$\mathrm{T}$ Terapeutas ocupacionais são freqüentemente requisitados a avaliar e tratar crianças com dificuldades variadas, relacionadas ao desenvolvimento da coordenação motora, capazes de interferir negativamente em seu desempenho ocupacional nos diferentes contextos em que se inserem (WILLOUGHBY; POLATAJKO, 1994). Desde o início do século passado, esforços têm sido empregados na tentativa de melhor definir e caracterizar tais dificuldades. Contudo, somente em 1994, em London, um grupo multidisciplinar de pesquisadores chegou a um consenso, através do estabelecimento do termo "transtorno do desenvolvimento da coordenação" (TDC), a fim de facilitar o progresso das pesquisas na área e desenvolver serviços baseados em evidências (MAGALHÃES et al., 2006).

De acordo com a $4^{\text {a }}$ edição revisada do Manual Estatístico e Diagnóstico de Distúrbios Mentais (DSMIV - APA, 2000), o TDC ocorre quando, na ausência de outros distúrbios físicos/neurológicos conhecidos, há um prejuízo acentuado no desenvolvimento das habilidades motoras (grossas e/ou finas), com desempenho nas atividades acadêmicas, de lazer e de vida diária significativamente abaixo do esperado para a idade cronológica e nível cognitivo da criança. A Classificação Estatística Internacional de Doenças e Problemas Relacionados à Saúde (CID-10 - OMS, 2000) adota termo - "transtorno específico do desenvolvimento da função motora" - e definição muito semelhantes à do DSM-IV, e estabelece que tal diagnóstico deva ser realizado após administração individualizada de testes estandardizados de coordenação motora. Esta nomenclatura, entretanto, é pouco utilizada em artigos científicos na área (MAGALHÃES et al., 2006).

Embora não haja dados relacionados à população brasileira, estima-se que a prevalência deste transtorno seja de 5 a $8 \%$ das crianças em idade escolar, com incidência maior no sexo masculino (DEWEY, 2002). Cermak et al. (2002) justificam que é difícil calcular com precisão a freqüência do TDC, pois não há definição de critérios objetivos de diagnóstico e outros fatores que influenciam as taxas de prevalência, tais como diferenças culturais e de métodos de avaliação. A despeito das controvérsias, sabe-se que as dificuldades motoras de crianças com TDC tendem a persistir na vida adulta com sérios comprometimentos no desempenho funcional de atividades significativas de vida diária como, por exemplo, dirigir carros (COUSINS;
SMITH, 2003; MAGALHÃES et al., 2004).

Diversos fatores, entre os quais predisposição genética, disfunção cerebral e complicações no parto têm sido investigados na tentativa de se esclarecer melhor a etiologia do transtorno, embora sem êxito até o momento (CERMAK et al., 2002). Em contrapartida, outros estudos confirmam a elevada freqüência do TDC associado a outras condições; sendo estimado que $68 \%$ das crianças com o transtorno apresentam diagnóstico múltiplo, principalmente em associação ao transtorno do déficit de atenção e hiperatividade (TDAH), distúrbios de aprendizagem (HENDERSON; HENDERSON, 2002) e problemas comportamentais diversos (IVERSEN et al., 2006).

Os sinais característicos do TDC incluem problemas de ritmo, de planejamento motor e na transferência de aprendizagem, coordenação motora pobre, tensão corporal, desajeitamento e declínio do desempenho com a repetição, dentre outros, o que torna bastante heterogêneo o grupo de crianças que apresentam esta condição (MAGALHÃES et al., 2004). Apesar dos sinais comumente apresentados serem motores, as conseqüências deste transtorno para crianças em idade escolar estendem-se para várias áreas do desempenho ocupacional, com impacto tanto na participação em atividades típicas da faixa etária (acadêmicas, de lazer e da vida diária) como na interação social e no senso de eficácia percebida.

De acordo com Cairney et al. (2005), crianças com TDC sofrem tanto no playground, onde estão sujeitas ao ridículo, como na sala de aula, onde as dificuldades motoras comprometem seu desempenho acadêmico, podendo conduzir a percepções reduzidas de sua competência pessoal. Alterações no senso de competência, por sua vez, podem gerar condutas de isolamento, reduzindo o repertório de atividades e agravando assim os sinais do transtorno.

Considerando tal contexto, o objetivo desta revisão é apontar a relação entre o desempenho motor e o senso de eficácia percebida de crianças com TDC em idade escolar, bem como suas possíveis implicações para a prática da Terapia Ocupacional.

\section{MÉTODOS}

Realizou-se levantamento bibliográfico das evidências disponíveis, na literatura estrangeira, sobre desempenho motor e senso de eficácia percebida em crianças com Transtorno do Desenvolvimento da Coordenação através de busca eletrônica nas bases de dados MEDLINE, OTDBase e OTseeker.

Para a discussão foram selecionados estudos origi- 
GALVÃO B. A. P. et al. Transtorno do desenvolvimento. Rev. Ter. Ocup. Univ. São Paulo, v.19, n. 1, p. 12-19, jan./abr. 2008.

nais (artigos experimentais), preferencialmente no idioma inglês, publicados no período de 1992 a 2007, utilizando os seguintes descritores: developmental coordination disorder, sense of self-efficacy, perceived self-efficacy, child and occupational therapy

\section{Auto-eficácia}

Considerados propriedade da Psicologia e tendo seu conceito originado da Teoria Cognitivo Social (BANDURA, 1986), os termos sinônimos auto-eficácia ou senso de eficácia percebida (variando conforme o autor) referem-se à crença pessoal em relação à capacidade do indivíduo em realizar determinada atividade (BANDURA, 1986). Tratase de uma avaliação ou percepção pessoal quanto à própria inteligência, habilidades e conhecimentos diversos, sendo que o foco principal não é possuir tais capacidades - não basta que estejam presentes; é necessário que o indivíduo acredite que as possua e nelas confie (BANDURA, 1977)

Neste contexto, três foram os parâmetros eleitos por Bandura (1977) para melhor caracterizar os termos em questão: magnitude, força e generalização. Magnitude corresponde ao nível de dificuldade inerente a atividade, enquanto força se refere ao grau que o indivíduo acredita ser capaz de realizar satisfatoriamente esta mesma atividade. E, por último, generalização, que diz respeito à capacidade de cada indivíduo em perceber sua auto-eficácia na atividade em foco e transferi-la para outras similares em diferentes ambientes (BANDURA, 1977).

Este conceito, entretanto, é comumente confundido com auto-estima, o que torna necessária sua diferenciação visando evitar possíveis interpretações equivocadas e, ao mesmo tempo, facilitar a compreensão do presente estudo. De acordo com o Dicionário Websester (1991), auto-estima se refere a confiança e satisfação em si próprio acrescida do senso de negativo/positivo (PATTERSON, 1991), enquanto a auto-eficácia trata da estimativa de quão bem o indivíduo pode desempenhar uma atividade específica, considerando sua experiência de vida e valores sociais da cultura em que se insere. Também tem sido sugerido que a auto-eficácia percebida pode explicar, parcialmente, a inconsistência freqüentemente observada por terapeutas ocupacionais entre as habilidades desenvolvidas por seus clientes e o uso destas fora do ambiente terapêutico (GAGE; POLATAJKO, 1994).

Uma importante diferença entre os conceitos de autoeficácia e auto-estima é o fato de o primeiro estar diretamente relacionado a um evento ou domínio específico da vida, enquanto o último é uma maneira relativamente estável na qual o indivíduo se percebe. A auto-estima é criada a partir da análise que a pessoa faz em relação a sua competência total, considerando seus fatores sociais relevantes (MAYBERRY, 1990). Por exemplo, um indivíduo se percebe competente em muitas atividades e mesmo assim pode ter uma baixa auto-estima, visto que estas atividades não são socialmente relevantes para ele. Inversamente, é possível apresentar uma baixa percepção da auto-eficácia em uma atividade e ainda assim possuir auto-estima elevada, pelo fato da auto-eficácia não ser determinante para a construção da auto-estima, embora contribua para tanto - conceitos complementares.

Ao correlacionar a percepção da auto-eficácia e o desempenho de atividades socialmente relevantes, Bandura (1977) constatou que elevada percepção da auto-eficácia em determinada atividade é crucial para um desempenho satisfatório. Isto porque altas expectativas de auto-eficácia resultam em maior empenho nas atividades - pessoas com este perfil estabelecem metas desafiadoras para si e regulam o esforço necessário para alcançá-las e superar possíveis obstáculos -, enquanto expectativas baixas de eficácia resultam na evasão das mesmas (BANDURA, 1986). Importante ressaltar também que o processo de seleção de atividades exerce efeito potencial no desenvolvimento humano, vez que é através da escolha da atividade que o individuo poderá ou não ter a oportunidade de desenvolver e aperfeiçoar suas habilidades, sejam elas físicas, cognitivas ou psico-sociais (GAGE; POLATAJKO, 1994).

\section{A RELAÇÃO ENTRE TDC E SENSO DE EFICÁCIA PERCEBIDA}

Cairney e colaboradores (2005), visando compreender melhor a relação existente entre o TDC e a atividade física reduzida com a influência mediadora da auto-eficácia generalizada, utilizaram como fonte os pares no ambiente escolar. Neste estudo de secção transversal, em uma amostra aleatória composta por 590 estudantes da $4^{\mathrm{a}}$ a $8^{\mathrm{a}}$ série de cinco escolas elementares em Ontário, Canadá, 7,5\% das crianças $(n=44)$ se encaixaram nos critérios diagnósticos para o transtorno. A proficiência motora da amostra foi avaliada com o formulário curto de teste Bruininks-Oseretsky (BRUININKS, 1978), a auto-eficácia generalizada com a escala de Auto-Percepção da Criança (HAY, 1992) e os níveis de atividade física foram obtidos a partir de um questionário de participação composto por 61 itens (CAIRNEY, 2005).

Embora os pesquisadores não tenham conseguido estabelecer com precisão os caminhos potenciais que relacionam a condição diagnóstica à atividade física reduzida (efeito não significativo), os resultados obtidos indicam que a auto- 
GALVÃO, B. A. P. et al. Transtorno do desenvolvimento. Rev. Ter. Ocup. Univ. São Paulo, v.19, n. 1, p. 12-19, jan./abr. 2008.

eficácia generalizada esclarece uma proporção considerável (28\%) da relação TDC - atividade física reduzida. Cairney et al. (2005) verificaram também que crianças com TDC são muito menos prováveis do que seus pares a participar do brincar vigoroso e atividades estruturadas, em função de não se perceberem como sendo suficientemente adequadas para atingir expectativas mínimas de desempenho, além de estarem mais propensas ao sedentarismo como estratégia de enfrentamento do risco de falha e humilhação.

$\mathrm{Na}$ tentativa de investigar com maior acuidade as manifestações específicas do TDC em crianças menores, Rodger e colaboradores (2003) se propuseram a avaliar seu impacto funcional através da utilização do Modelo de Avaliação de Desfechos Motores (MAOM) (COSTER; HALEY, 1992) numa amostra de 20 crianças australianas (12 meninos e 08 meninas) de 04 a 08 anos. Todas as crianças foram filmadas enquanto realizavam o Visual Motor Impairment (VMI) (BEERY; BUKTENICA, 1997) e uma série de tarefas de recorte e escrita. O Inventário de Avaliação Pediátrica de Incapacidade (PEDI) (HALEY et al., 1992), a Escala Pictórica de Competência Percebida e Aceitação Social (PCSA) (HARTER \& PIKE, 1983), o MAOM e a Escala de Desenvolvimento Motor (PDMS) (FOLIO; FEWELL, 1983) também foram utilizados.

Uma das questões apresentadas pelo autor visa verificar se as dificuldades acadêmicas e na vida diária são realmente manifestações do TDC. Para datar o impacto funcional, este transtorno tem sido documentado na literatura em relação à diminuição do lazer e participação no brincar, além do impacto negativo na confidência da criança em habilidades físicas, sociais e no seu bem-estar emocional. Segundo Rodger e colaboradores (2003), manifestações mais específicas não têm sido documentadas e a maioria das pesquisas tem sido conduzida com crianças de 07 a 12 anos com poucos estudos envolvendo crianças mais novas.

Os resultados apontam que crianças com TDC têm dificuldades significativas com o uso de estratégias de recorte (posição inicial da tesoura) e preensão do papel, conforme ficou evidenciado na observação das filmagens. Em relação à participação, as crianças se perceberam como mais competentes tanto na escala de competência física e cognitiva, como na aceitação materna e de seus pares. Tais achados estão em contraste com outros estudos de crianças mais velhas com transtorno do desenvolvimento da coordenação. Segundo os autores, há várias razões para estes achados: 1) crianças mais novas podem estar fornecendo respostas socialmente desejáveis em seu auto-relato; 2) crianças mais novas podem não ser capazes de discriminar respostas potenciais como as mais velhas, e tendem a responder em direção aos extremos da escala; e por último, 3) crianças mais novas podem ainda não ter começado a se comparar com seus pares (RODGER et al., 2003).

Schunk (1989) corrobora tais razões afirmando que a auto-eficácia é um processo evolutivo e crianças mais novas percebem seus esforços conduzindo a resultados diretos, enquanto que, com o avançar da idade, o conceito de habilidade emerge associado à avaliação em relação aos outros (aos pares principalmente). É possível que crianças mais novas com TDC se percebam "dando duro" nas tarefas, mesmo quando estas são difíceis. Se elas atribuírem esforço à competência, elas se perceberão como sendo competentes devido unicamente a seus esforços (RODGER et al., 2003) - o que não ocorre em amostras de crianças com média de idade acima de 09 anos (MISSIUNA; POLLOCK, 1995). Assim, segundo Schunk (1989), esta pode ser a época perfeita para intervir com esta população a fim de melhorar suas habilidades motoras e reforçar sua auto-eficácia, vez que tal crença influencia diretamente no esforço e persistência. Em outro estudo, ao revisar a Teoria Cognitivo Social (BANDURA, 1986) e seus princípios para o aprendizado, Schunk (1996) propõe que, embora elevada auto-eficácia não produza desempenho competente quando as capacidades e o conhecimento estão ausentes, tal senso é benéfico porque motiva o indivíduo a melhorar sua competência. Expectativas finais ou crenças relacionadas aos prováveis resultados são importantes porque os indivíduos se engajam em atividades nas quais eles acreditam terem resultados positivos. Desta forma, os julgamentos de auto-eficácia atuam como mediadores entre as reais capacidades (aptidões, conhecimentos e habilidades) e a própria performance. Neste contexto, pais, professores e profissionais da área da saúde exercem papel decisivo, pois, segundo o autor ressalta, $f e-$ edback positivo melhora a crença pessoal, incentivando as crianças a se engajarem em novas atividades, apesar deste aumento poder ser temporário se os esforços subseqüentes forem escassos (SCHUNK, 1996).

Segundo Mandich et al (2003), os pais observam e relatam que a participação bem sucedida constrói confiança em seus filhos, o que permite a eles tentar brincar de outras atividades. Estudos recentes apontam para a influência positiva da participação da criança em atividades típicas da infância na saúde, auto-estima e ajustamento social (KLEIBER, 1999; LARSON, 2000). Contudo, no caso das crianças com TDC, tal participação geralmente está restrita em função das dificuldades motoras por elas apresentadas (MANDICH et al., 2003).

Em pesquisa qualitativa utilizando entrevistas semi-estruturadas com 10 famílias de crianças com TDC, Mandich et al. (2003) objetivaram explorar o impacto do transtorno na vida desta população a partir da perspectiva 
GALVÃO B. A. P. et al. Transtorno do desenvolvimento. Rev. Ter. Ocup. Univ. São Paulo, v.19, n. 1, p. 12-19, jan./abr. 2008.

de seus pais. A amostra utilizada foi composta por 12 pais e mães de crianças com idades entre 07 e 12 anos, sendo 09 do sexo masculino e 01 do feminino, todas clientes da clínica universitária (Universidade de Toronto, Canadá), cujo tratamento ou foi finalizado ou estava no estágio final.

A partir deste estudo, os pesquisadores identificaram consideráveis restrições nas atividades de base motora, corroborando Missiuna e Pollock (1995), e algumas conseqüências negativas, tais como reduzida percepção da competência, baixa auto estima e restrição na participação social destas crianças. Entretanto, foi possível identificar no decorrer do estudo que, com a realização do tratamento terapêutico ocupacional e suporte familiar, tal situação é passível de ser revertida, alimentando o sentimento de capacidade e eficiência e melhorando a auto-estima dos portadores do transtorno (MANDICH et al., 2003).

Dando continuidade a análise da percepção da competência física entre os portadores de TDC, Dunn e colaboradores (1994), visando explorar a relação existente entre a auto-percepção da competência física e o comportamento das crianças, realizaram um estudo de análise regressiva envolvendo 195 estudantes de $3^{\circ}$ a $6^{\circ}$ série, sendo 97 meninos e 98 meninas. A fim de medir a severidade da coordenação motora foi utilizado o teste Test of Motor Impairment - Henderson Revision (TOMI) (STOTT, MOYES; HENDERSON, 1984); para avaliar a percepção da competência física o The Atletic Competence Subscale of The Self Perception Profile for Children (Harter, 1985), a importância da competência física foi medida através dos itens correspondentes da escala Importance Rating Scale of Harter's Self Perception Profile for Children (HARTER, 1985); enquanto, para respostas de habilidades sociais, recorreu-se ao The Children's Social Desirability Scale (CRANDALL et al., 1965).

Embora a literatura relate que crianças com TDC desenvolvam, com o avançar da idade, baixas percepções de competência física resultando na recusa de se envolverem em atividades motoras, o presente estudo encontrou crianças, principalmente da $5^{\circ}$ e $6^{\circ}$ série, com altas percepções da competência mesmo quando aumentado o grau de dificuldade das atividades motoras específicas da faixa etária (DUNN et al, 1994). Acontecimento explicado pelos autores de duas formas: a primeira refere-se à escolha das crianças ao se comparar com pares de desempenho semelhante ou inferior a si próprio, e a segunda referente ao enorme esforço desempenhado nas atividades motoras correspondendo, assim, a altas percepções de competência física. Tal achado é contrastante ao de Rodger e colaboradores (2003), os quais verificaram elevada percepção de competência apenas em amostras de crianças mais novas (04 a 08 anos). Neste senti- do, constata-se a carência de novas pesquisas e a necessidade de cautela em relação às generalizações, principalmente no que diz respeito à população brasileira.

Indivíduos com TDC formam um grupo bastante heterogêneo: enquanto alguns apresentam comprometimento apenas nas habilidades motoras (finas e/ou grossas), outros possuem limitações em diferentes componentes de desempenho. Podendo esta variedade estar relacionada ao sexo e a idade, Piek et al. (2006) realizou um estudo, na tentativa de averiguar o impacto das habilidades motoras na autopercepção de crianças e adolescentes dos sexos masculino e feminino. Um total de 265 crianças e adolescentes foram incluídas no estudo, sendo divididas em dois grupos: (1) 164 crianças, sendo 80 meninas e 84 meninos com idade entre 7 e 11 anos; e (2) 101 adolescentes, sendo 64 meninas e 37 meninos com idade entre 12 e 15 anos.

$\mathrm{Na}$ avaliação do desenvolvimento motor foi utilizado o McCarron Assrssment of Neuromuscular Development (MAND) (MCCARRON, 1982), enquanto a auto-percepção foi medida através do Self Perception Profile for Children (HARTER, 1985) e Self Perception Profile for Adolescents (HARTER, 1988). Os resultados encontrados demonstraram grande impacto na percepção da competência atlética e da competência escolar quando considerada a auto-percepção e o nível de habilidade motora dos participantes. O argumento de que a presença de baixos resultados emocionais e sociais estão relacionados a um precário desempenho motor é sustentado pelo fato de meninos e meninas com TDC considerarem a competência atlética como contribuinte essencial para o sentimento de auto-valor (PIEK et al., 2006). Tal achado, segundo Piek e colaboradores (2006), é mais evidente no grupo do sexo feminino, o qual possui percepções reduzidas mais acentuadas que os integrantes do grupo masculino.

A despeito do crescente interesse e uso pela Teoria Cognitivo Social (BANDURA,1986) no ambiente clínico e científico, questões sobre quando e como a auto-eficácia se desenvolve, se ela se opera uniformemente através dos domínios da vida e quais são os caminhos aceitáveis para avaliá-la ainda persistem. Neste sentido, o trabalho de Pastorelli et al. (2001) sobre a estrutura da auto-eficácia percebida em crianças emerge como importante recurso a fim de apresentar algumas considerações a respeito das relações existentes entre o indivíduo e seu ambiente na construção e desenvolvimento das crenças pessoais.

Durante as primeiras fases da vida, a família exerce um papel crucial na aquisição de conhecimento sobre si mesmo e o ambiente externo, vez que é a primeira fonte de informação dos valores sociais para a criança (PASTORELLI et al., 2001). m função desta dependência, elas 
GALVÃO, B. A. P. et al. Transtorno do desenvolvimento. Rev. Ter. Ocup. Univ. São Paulo, v.19, n. 1, p. 12-19, jan./abr. 2008.

rapidamente aprendem como influenciar o comportamento de seus cuidadores através de ações bem sucedidas, configurando assim o início do desenvolvimento dos modelos de eficácia percebida (BANDURA, 1986). Segundo Pastorelli (2001), os pares são a segunda fonte de informação para estas crianças, contribuindo para a formação da autoeficácia através de observações e comparações individuais. Já no ambiente escolar, elas experimentam novas relações e desafios que podem ampliar e validar suas habilidades, tendo os professores papel essencial no amadurecimento do senso de auto-eficácia. A percepção das crianças sobre suas capacidades é significativamente influenciada pelo modo como os professores avaliam seu desempenho e os ajudam a aperfeiçoar ou desenvolver habilidades de auto-regulação no gerenciamento das demandas apresentadas pelo ambiente em que se inserem.

Considerando a hipótese de Bandura (1977, 1986) sobre a auto-eficácia afetar a escolha de atividades, o esforço e a persistência, verifica-se que os indivíduos que possuem baixo senso de auto-eficácia para realizar uma tarefa podem evitá-la, enquanto aqueles que acreditam serem capazes participam mais prontamente. Especialmente quando encontram dificuldades, os que acreditam serem capazes de desempenhar bem trabalham duro e persistem mais do que os que duvidam de suas capacidades. Crianças em idade escolar adquirem informações para avaliar a auto-eficácia a partir do seu desempenho, experiências de observações, formas de persuasão e reações psicológicas (PASTORELLI et al., 2001).

Tais afirmações puderam ser confirmadas a partir da utilização da Escala de Auto-Eficácia Percebida (CPSE), um conjunto multidimensional de escalas desenvolvido por Bandura (1977), em amostra composta por 1180 crianças provenientes de três países europeus (Itália, Hungria e Polônia). Os resultados indicam que a eficácia auto-regulatória é definida diferentemente, especialmente na amostra húngara, em função do comportamento de dois itens: item 25 - resistir à pressão dos pares, e item 26 - permanecer firme quando alguém convida para fazer algo inconveniente (PASTORELLI et al., 2001).

Os achados deste estudo suportam a idéia de que a percepção das crianças guia suas ações ocupacionais em direção congruente a suas crenças de eficácia social, auto-regulatória e acadêmica, sendo que os modelos de crenças de eficácia pessoal diferem através das origens. Embora futuras pesquisas sejam necessárias para expandir o tamanho da amostra e o número de ítens representando a eficácia auto-regulatória, tal análise revelou também efeito significativo de gênero: meninos expressaram menor eficácia para resistir a pressão dos pares em se engajar em atividades transgressivas do que as meninas (PASTORELLI et al., 2001).

\section{CONSIDERAÇÕES FINAIS}

Em sua prática clínica, terapeutas ocupacionais, algumas vezes, observam uma discrepância alarmante entre as habilidades de desempenho ocupacional desenvolvidas no ambiente terapêutico e o direcionamento a elas conferido em outros espaços (GAGE; POLATAJKO, 1994). A literatura sugere que a auto-eficácia percebida possa explicar parcialmente esta discrepância; contudo, uma compreensão de tal conceito ainda não foi integrada à prática da Terapia Ocupacional. Tanto a ausência de consciência do construto como a falta de habilidade para avaliá-lo são responsáveis por esta significativa lacuna.

Neste sentido, algumas escalas têm sido desenvolvidas como ferramentas de avaliação para clientes que experimentam disfunções no seu desempenho ocupacional. Dentre elas, a Self-Efficacy Gauge ou The Gauge (GAGE et al, 1990) se destaca por ter sido criada especialmente para terapeutas ocupacionais. Tal instrumento foi desenvolvido objetivando mensurar o nível de eficácia percebida em uma ampla variedade de atividades nas diferentes áreas de desempenho, assim como sua variação ao longo do tempo (GAGE et al., 1990).

Este tipo de informação permite aos terapeutas saber se seus clientes acreditam ou não serem capazes de desenvolver determinadas habilidades, e, assim, escolher metas de tratamento mais realistas (GAGE et al., 1990). Segundo Gage e Polatajko (1994), terapeutas devem compreender que a aquisição de uma habilidade, na ausência da crença pessoal em desempenhá-la, não é suficiente para melhorar o desempenho ocupacional a despeito dos esforços empreendidos pelo profissional responsável.

Desta forma, considerando as dificuldades características do TDC, o desenvolvimento de estratégias envolvendo a adequação das expectativas de pais e professores a níveis razoáveis, e a seleção de atividades nas quais as crianças possam obter sucesso e lhes sejam relevantes parece apropriado. Este processo pode ocorrer somente se os indivíduos envolvidos reconhecerem as limitações existentes e se dispuserem, de fato, a analisar e modificar as condições adversas. Isto, pois freqüentemente esta população é encarada como desajeitada e/ou preguiçosa, a despeito de suas reais dificuldades, e avisada para trabalhar duro, o que reforça seu fracasso e baixo senso de auto-eficácia (GAGE; POLATJAKO, 1994).

O conhecimento dos mecanismos de funcionamento do transtorno do desenvolvimento da coordenação aliado a compreensão da importância do senso de eficácia 
percebida confere, aos terapeutas ocupacionais, condições de auxiliar crianças com TDC e seus familiares a conviver de maneira mais satisfatória com as limitações existentes, construindo um senso de auto-eficácia adequado e participando das atividades típicas da infância que lhes sejam significativas.

GALVÃO, B. A. P.; LAGE, N. V.; RODRIGUES, A. A. C. Developmental coordination disorder and sense of self-efficacy: implications for occupational therapy's practice. Rev. Ter. Ocup. Univ. São Paulo, v.19, n. 1, p. 12-19, jan./abr. 2008.

\begin{abstract}
Developmental Coordination Disorder (DCD) is characterized by a marked deficit in the development of children's motor skills (gross and/or fine), with impact in the participation in typical activities of the childhood. Consequences of the disorder include restriction in social interaction and low sense of self-efficacy. Although occupational therapists are able to evaluate and treat children with this profile, they don't seem to know the importance of the sense of self-efficacy, as well as its assessment tools. In this way, this review points to the relation between the motor performance and the sense of efficacy of children with DCD in school age, as well as its possible implications for Occupational Therapy's practice.
\end{abstract}

KEY WORDS: Motor skills disorders. Self efficacy. Child. Psychomotor performance. Occupational therapy.

\title{
REFERÊNCIAS
}

1. AMERICAN PSYCHIATRIC ASSOCIATION. Diagnostic and statistical manual of mental disorders. $4^{\mathrm{a}}$ ed revised. Washington: APA, 2000.

2. BANDURA, A. Self-efficacy: toward a unifying theory of behavioral change. Psychological Review, v. 84, n. 2, p. 191-215, 1977.

3. BANDURA, A. Social foundations of thought and action - a social cognitive theory. Englewood Cliffs, NJ: Prentice Hall, 1986. In.: BANDURA, A. Self-efficacy: toward a unifying theory of behavioral change. Psychological Review, v. 84, n. 2, p. 191-215, 1977.

4. BEERY, K. E.; BUKTENICA, N. A. Developmental test of visual motor integration. Austin, TX: Proed, 1997.

5. BRUININKS, R. H. Bruininks-Oseretsky test of motor proeficiency: examiner's manual. Circle Pines, MN: American Guidance Service, 1978. In.: CAIRNEY, J. et al. Developmental coordination disorder, generalized self-efficacy toward physical activity and participation in organized free play activities. Journal Pediatrics, 147, p .515-520, 2005.

6. CAIRNEY, J. et al. Developmental coordination disorder, generalized self-efficacy toward physical activity and participation in organized free play activities. Journal Pediatrics, 147, p. 515-520, 2005.

7. CERMAK, S.A.; GUBBAY, S.S.; LARKIN, D. What is developmental coordination disorder? In: CERMAK, S.A.; LARKIN, D. Developmental coordination disorder. Albany, NY: Delmar, 2002. Cap. 1, p. 2-22.

8. COUSINS, M.; SMYTH, M. M. Developmental coordination impairments in adulthood. Human Movement Science, v. 22, p. 433-459, 2003.

9. COSTER, W.; HALEY, S. Conceptualization and measurement of disablement in infants and young children. Infants and Young Children, v. 4, p. 11-22, 1992.

10. DAUB, M. Prenatal development through mid-adult-hood. In: HOPKINS, H. L; SMITH, H. D (eds.). Willard and Spackman's occupational therapy. 7 th. ed. Philadelphia: Lippincott, 1988. p. 50-75.

11. DEWEY, D. Subtypes of developmental coordination disorder. In: CERMAK, S.A., LARKIN, D. Developmental coordination disorder. Delmar: Thonson Learning, 2002.

12. DUNN, J. L. C.; WATKINSON, E. J. A study of the relationship between physical awkwardness and children's 
GALVÃO, B. A. P. et al. Transtorno do desenvolvimento. Rev. Ter. Ocup. Univ. São Paulo, v.19, n. 1, p. 12-19, jan./abr. 2008.

perceptions of physical competence. Adapeted Physical Activity Quarterly, p. 275-283, 1994.

13. FOLIO, M. R.; FEWELL, R. R. Peabody developmental motor scales and activity cards. Hingham, M. A: DLM Teaching Resources, 1983.

14. GAGE, M.; NOH, S.; POLATAJKO, H. J.; KASPAR, V. Measuring perceived self-efficacy in Occupational Therapy. American Journal Occupational Therapy., v. 48, n. 9, p. 783-790, 1990.

15. GAGE, M.; POLATAJKO, H. Enhancing occupational performance through an understanding of perceived selfefficacy. American Journal Occupational Therapy, n. 48, p. 452-461, 1994.

16. HALEY, S. M. et al. Pediatric evaluation of disability inventory. Boston, MA: New England Medical Centre, 1992.

17. HARTER, S.; PIKE, R.. The pictorial scale of perceived competence and social acceptance for youngchildren. Denver, C. O: University of Denver, 1983. In.: RODGER, S. et al. Motor and functional skills of children with developmental coordination disorder: a pilot investigation of measurement issues. Human Movement Science, v. 22, p. 461-478, 2003.

18. HAY, J. A. Adequacy in and predilection for physical activity in children. Clinical Journal of Sport Med, 2, p.192-201, 1992. In:: CAIRNEY, J. et al. Developmental coordination disorder, generalized self-efficacy toward physical activity and participation in organized free play activities. Journal Pediatrics, v. 147, p. 515-520, 2005.

19. HENDERSON, S. E.; HENDERSON, L. Toward an understanding of developmental coordination disorder: terminological and diagnostic issues. Adapted Physical Activity Quarterly, v. 19, p. 11-31, 2002.

20. IVERSEN, S.; KNIVSBERG, A.M.; ELLERTSEN, B.; NODLAND, M.; LARSEN, T.B. Motor coordination in 5-6-year-old children with severe behavioral and emotional problems. Emotional Behav. Difficulties, v. 11, n. 3, p. 169-185, 2006.
21. MAGALHÃES, L. C.; NASCIMENTO, V. C. S.; REZENDE, M. B. Avaliação da coordenação e destreza motora ACOORDEM: etapas de criação e perspectivas de validação. Revita de Terapia Ocupacional da Universidade de São Paulo, v. 15, n. 1, p. 17-25, 2004.

22. MAGAlhães, L. C., MissiunA, C.; WONG, S. Terminology used in research reports of developmental coordination disorder. Developmental Medicine and Child Neurology, v. 48, p. 937-941, 2006.

23. MANDICH, A. D.; POLATAJKO, H. J.; RODGER, S. Rites of passage: understanding participation of children with developmental coordination disorder. Human Movement Science, v. 22, p. 583-595, 2003.

24. MAYBERRY, W. Self esteem in children: considerations for measurement and intervention. American Journal Occupational Therapy, n. 44, p.. 729-734, 1990.

25. PASTORELLI, C.; CAPRARA, G. V.; BARBARANELLI, C.; ROLA, J.; ROZSA, S.; BANDURA, A. The structure of children's perceived self-efficacy: a cross-national study. European Journal Psychologic Assessment, v. 17, n. 2, p 87-97, 2001.

26. PATTERSON, R. F. New Webster's Dictionary. Miami, 1991. Disponível em: http://www.websters-online-dictionary. org. Acesso em fev. 2007.

27. PIEK, J. P.; GAYMAN, G. B.; BARRETT, N. C. The relationship between fine and gross motor ability, selfperceptions and self-worth in children and adolescents. Human Movement Science, p. 65-75, 2006.

28. RODGER, S. et al. Motor and functional skills of children with developmental coordination disorder: a pilot investigation of measurement issues. Human Movement Science, v. 22, p. 461-478, 2003.

29. WILLOUGHBY, C.; POLATAJKO, H. J. Motor problems in children with developmental coordination disorder: review ok the literature. American Journal Occupational Therapy, v. 49, n. 8, p. 787-794, 1994. 